OPEN ACCESS

Edited by:

Jake Patterson,

Sheffield Teaching Hospitals NHS

Foundation Trust, United Kingdom

Reviewed by:

Volkan Ulker,

Medicana International Izmir Hospital,

Turkey

Dirk Lange,

University of British Columbia, Canada

Takaaki Inoue,

Kobe University, Japan

Kevan Sternberg,

University of Vermont, United States

*Correspondence:

Shaokai Zheng

shaokai.zheng@outlook.com

Specialty section:

This article was submitted to

Endourology,

a section of the journal

Frontiers in Urology

Received: 15 December 2021 Accepted: 06 January 2022

Published: 26 January 2022

Citation:

Zheng S, Amado P, Kiss B, Stangl F, Haeberlin A, Sidler D,

Obrist D, Burkhard F and Clavica F

(2022) Quantitative Evaluation

of Encrustations in Double-J

Ureteral Stents With Micro-

Computed Tomography and

Semantic Segmentation.

Front. Urol. 2:836563

doi: $10.3389 /$ fruro.2022.836563

\section{Quantitative Evaluation of Encrustations in Double-J Ureteral Stents With Micro-Computed Tomography and Semantic Segmentation}

\author{
Shaokai Zheng ${ }^{1 *}$, Pedro Amado ${ }^{1}$, Bernhard Kiss ${ }^{2}$, Fabian Stangl ${ }^{2,3}$, Andreas Haeberlin $^{4}$, \\ Daniel Sidler ${ }^{5}$, Dominik Obrist ${ }^{1}$, Fiona Burkhard ${ }^{2}$ and Francesco Clavica ${ }^{1}$ \\ ${ }^{1}$ ARTORG Center for Biomedical Engineering Research, University of Bern, Bern, Switzerland, ${ }^{2}$ Department of Urology, \\ Inselspital, Bern University Hospital, University of Bern, Bern, Switzerland, ${ }^{3}$ Department of Urology, Cantonal Hospital Olten, \\ Olten, Switzerland, ${ }^{4}$ Department of Cardiology, Inselspital, Bern University Hospital, University of Bern, Bern, Switzerland, \\ ${ }^{5}$ Department of Nephrology and Hypertension, Inselspital, Bern University Hospital, University of Bern, Bern, Switzerland
}

Accurate evaluation of stent encrustation patterns, such as volume distribution, from different patient groups are valuable for clinical management and the development of better stents. This study quantitatively compares stent encrustation patterns from stone and kidney transplant patients. Twenty-seven double-J ureteral stents were collected from patients with stone disease or who underwent kidney transplantation. Encrustations on stent samples were quantified by means of micro-Computed Tomography and semantic segmentation using a Convolutional Neural Network model. Luminal encrustation volume per stent unit was derived to represent encrustation level, which did not differ between patient groups in the first six weeks. However, stone patients showed higher encrustation levels over prolonged indwelling times $(p=0.02)$. Along the stent shaft body, the stone group showed higher encrustation levels near the ureteropelvic junction compared to the ureterovesical junction ( $p=0.013$ ), whereas the transplant group showed no such difference. Possible explanations were discussed regarding vesicoureteral reflux. In both patient groups, stent pigtails were more susceptible to encrustations, and no difference between renal and bladder pigtail was identified. The segmentation method presented in this study is also applicable to other image analysis tasks in urology.

Keywords: Double J, ureteral stent, encrustation, stone, renal transplantation, micro CT, segmentation, deep learning

\section{INTRODUCTION}

Double-J ureteral stents are commonly used to bypass obstruction and alleviate pain in acute obstruction as well as in preparations prior to endoscopic stone treatment, or to stent the ureterovesical anastomosis after kidney transplantation to avoid obstruction due to edema in the early postoperative phase. In spite of various material upgrades and design modifications, 
encrustation remains a major problem causing stent associated complications with a significant impact on patients' quality of life $(1,2)$. Encrusted stents can become blocked and cause obstructive pyelonephritis. Foreign bodies in the urinary tract also tend to be colonized by bacteria that cause urinary tract infections. Severely encrusted stents can no longer be retrieved endoscopically and require more invasive approaches to be removed. As a result, indwelling stents have to be replaced in regular intervals. For stone patients, indwelling times longer than six weeks have been associated with significantly higher encrustation rates (2-4). For transplant patients, indwelling times from two to six weeks after transplantation have been recommended based on urinary tract infection (UTI) rates and stent-related complications such as pain, hematuria, encrustation and migration (5-8).

In this study, we are interested in clarifying the encrustation level over indwelling time in both stone and transplant patient groups, with a specific focus on the localization of the encrustations in each group. Therefore, evaluation of the encrustation volume is crucial. Earlier approaches were mainly qualitative, relying on visual examination of Scanning Electron Microscopy (9) or kidneyureter-bladder (KUB) radiography images $(10,11)$. Extracting quantitative information from KUB images had been attempted by measuring the projected area of encrustations (12). Unfortunately, the inherent uncertainty is not negligible as encrustations are threedimensional. Another established approach proposed to measure the level of encrustation by weighing the stent sample before and after oxidative acid treatment to dissolve the encrustations (13). The spatial distribution of encrustations, however, is destroyed during the process, and encrustations in the stent lumen (luminal encrustations) and on the external surfaces cannot be distinguished. In addition, encrustations on external stent surfaces can be affected during stent removal and consequently introduce significant uncertainties to quantitative results. It is therefore desirable to isolate the luminal encrustations, which are less affected by stent removal and critical to the drainage capacity of indwelling stents.

In a recent publication, micro-computed tomography ( $\mu$-CT) was applied to quantify stent encrustation volumes in order to assess the anti-encrustation efficacy of two commercially available stents (14). The authors performed morphological segmentation on the $\mu$-CT images, and managed to distinguish between luminal and external encrustations. Based on their results, more than $90 \%$ of the stent had luminal encrustations, which appeared more in the shaft body (the straight part of the stent) than in the renal and bladder pigtails, respectively. Nonetheless, one limitation associated with the method was that the volume of the individual stent remained unknown, so the encrustation volume might be biased according to the actual stent material volumes. Moreover, treating the entire stent shaft body as a unified section inherently ignores any heterogeneity of the encrustation volume distribution along the shaft, which might overlook some key characteristics along the shaft body.

In this study, we compared patterns of luminal stent encrustation volumes in stone and transplant patients. A semantic segmentation approach was first evaluated and implemented by means of Convolutional Neural Network (CNN) to simultaneously quantify the volume of luminal encrustations and of the stent. Luminal encrustation volume per stent unit was derived, representing the normalized encrustation level, such that the inter-subject changes of stent volume were accounted for. Further, luminal encrustation levels along the stent were assessed to evaluate the heterogeneity along the stent, and comparisons were made between stone and transplant patients.

\section{MATERIALS AND METHODS}

The retrospective study is reported in accordance with guidelines from the STROBE statement (15). The stone and transplant cohorts consisted of double-J stents removed endoscopically from patients who underwent stone treatment and kidney transplantation, respectively, between January 2020 and June 2021 at the Department of Urology of Bern University Hospital $(\mathrm{n}=24)$ and the Cantonal Hospital Olten $(\mathrm{n}=3)$ in Switzerland. Samples were selected by matching age, gender, presence of UTI, and stent indwelling time were matched between the two cohorts $(\mathrm{p}>0.05)$. Stents placed for external obstructions such as pregnancy and urothelial carcinoma, and stents with unknown indwelling times were excluded.

All stents were collected as by-products of regular urological treatment and the personally identifiable information were anonymized. Under the Human Research Act (Swiss Federal Act on Research involving Human Beings, Art. 2, The Federal Assembly of the Swiss Confederation), approval of the local ethics committee was exempted, and informed consent was waived. Written general consent was obtained from all patients. All methods were carried out in accordance with relevant guidelines and regulations.

Each collected stent was dried in an oven at $60^{\circ} \mathrm{C}$ for three hours to remove residual urine, which helps solidify the sample and prevent contamination during the following processes. To assess the encrustation volume in different sections of the stent, four sections of each stent were separated, i.e., the renal pigtail, the proximal straight part (near the ureteropelvic junction), the distal straight part (near the ureterovesical junction), and the bladder pigtail (see Figure 1A). This was done under the assumption that the two junctions are critical regions for the development of encrustations as they are the entrance and exit of the ureter, where urine flows are regulated by the physiologically narrowing tract.

Subsequently, each section was scanned using a $\mu$-CT scanner (SCANCO Medical AG, Bruettisellen, CH) operated at $90 \mathrm{kVp}$ and $200 \mu \mathrm{A}$ with an integration time of $200 \mathrm{~ms}$, optimized based on preliminary experiments. The final resolution was $11.4 \mu \mathrm{m}$ in all three dimensions. The acquired images were segmented using a CNN model known as the U-Net (16), available in the Dragonfly software (v2020.1, Object Research Systems Inc. Quebec, CA). The segmentation results allowed evaluation of the luminal encrustations including the luminal space of the side holes ( $\mathrm{SHs}$ ) without losing their spatial distribution (see Figures 1B-E), thus offering a more reliable representation of the encrustations. Full technical details on $\mu$-CT, discussion on accuracy, and examples can be found in the Supplementary Material. 


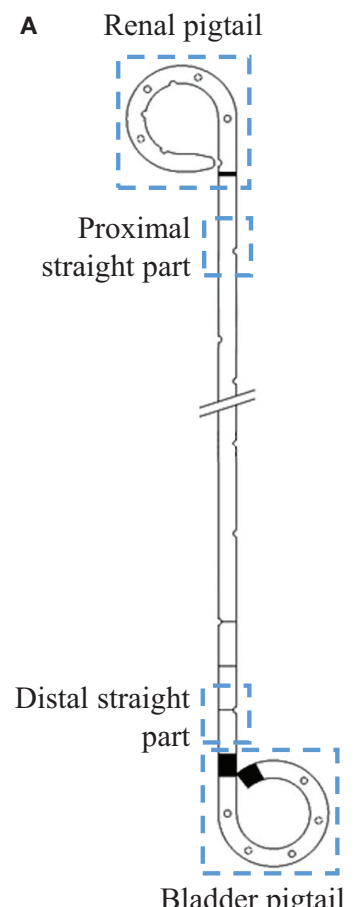

B

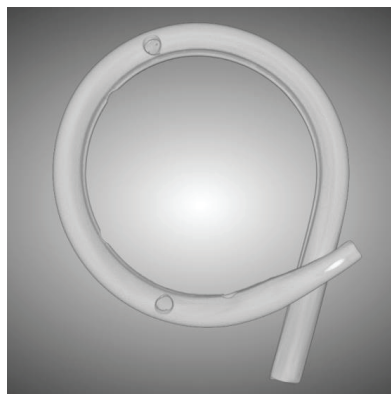

C

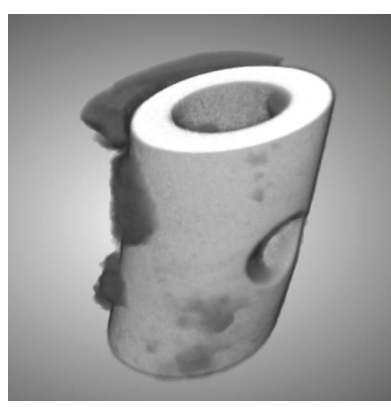

D

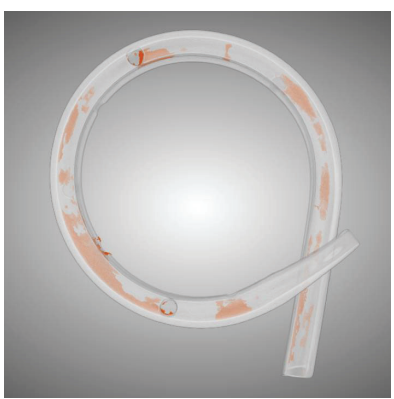

$\mathbf{E}$

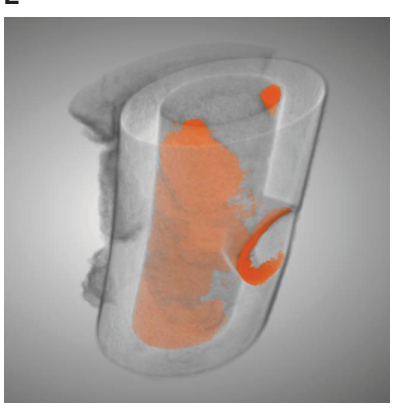

FIGURE 1 | lllustration of the four separated sections from a ureteral stent (A). Three dimensional $\mu$-CT images from the pigtail and straight part before (B, C) and after (D, E) segmentations, respectively. Luminal encrustations from the segmentation results are shown in orange, where stents are rendered semi-transparent for better visualization.

After the segmentation, volumes of the encrustations and of the stent were extracted, respectively. By defining the encrustation volume ratio (EVR) as the encrustation volume normalized by the corresponding stent volume, which gives the encrustation volume per stent unit, the bias introduced by the different volumes of individual stent samples was eliminated. The total encrustation volume ratio (TEVR) was defined by summing the EVR over the four stent sections, which indicates the overall susceptibility to encrustations of a stent. To study the relative level of encrustations at different stent sections, we defined the encrustation risk level (ERL) by dividing EVR over TEVR. As such, the inter-subject variability between samples is removed, as the ERL of each stent sums to one (100\%). The stent section with highest ERL would be likely to attract more encrustations.

To compare the encrustation level over time, stents from stone patients were divided into two groups: group one with indwelling time $<42 \mathrm{~d}$, and group two with indwelling time $\geq 42$ d. The choice of 42 days was based on the fact that an indwelling time over six weeks is commonly associated with significantly higher encrustation levels (2-4). Since optimal removal time in transplant patients has been reported to range between two and six weeks and no consensus has been reached, subgroups with indwelling times $<28 \mathrm{~d}$ or $\geq 28 \mathrm{~d}$ (four weeks) were chosen for comparison.

For statistical comparisons, the two-sided Mann-Whitney Utest was used for continuous data, while the Fisher's exact test was used for the categorical data in Table 1. P-values from multiple comparisons were corrected using the Bonferroni-Holm method, and $\mathrm{p}<0.05$ was considered significant in this study.

\section{RESULTS}

A total of 27 stents were analyzed, as summarized in Table $\mathbf{1 .}$ Stent indwelling times were 11-99 days for stone patients and 2247 days for kidney transplant patients, with no significant difference between the groups $(\mathrm{p}=0.4)$. All retrieved stents were made of polyurethane manufactured by PURE Medical Device SA (Geneva, CH) and Optimed Medical Instruments $\mathrm{GmbH}$ (Ettlingen, DE).

\section{Encrustations Near Side Holes}

Segmented images from stents at different indwelling times are shown in Figure 2 for each patient group. The amount of luminal encrustations (orange) in the stone group seems to increase over time, which is not observed in the transplant group. The increasing encrustation in the stone group is more apparent on the renal pigtail and proximal straight part of the stent. Aggregates of encrustations were mostly found near the SHs, whose locations along the pigtails are marked (arrows) in Figure 2. The example with indwelling time of 90 days showed complete blockage in the proximal lumen, as evidenced by the cross-sectional inset in Figure 2. 
TABLE 1 | Characteristics of patients and stents.

\begin{tabular}{|c|c|c|c|}
\hline & Stone patients & Transplant patients & $p$ value \\
\hline Patients, $n$ & 16 & 10 & \\
\hline Female, $n(\%)$ & 6 (37.5\%) & 5 (50\%) & 0.7 \\
\hline Median age, yr (IQR) & $60.5(48.5-75)$ & 57 (40-73) & 0.7 \\
\hline Presence of UTI, $n$ (\%) & 3/16 (18.8\%) & $4 / 10(40 \%)$ & 0.4 \\
\hline Ureter units, $n$ & 17 & 10 & \\
\hline \multicolumn{4}{|l|}{ Stone location, $n$ (\%) } \\
\hline Nephrolithiasis & $10 / 17$ (58.8\%) & NA & \\
\hline Ureterolithiasis & 6/17 (35.3\%) & NA & \\
\hline Nephro- \& Ureterolithiasis & $1 / 17$ (5.9\%) & NA & \\
\hline Collected stents, $n$ & 17 & 10 & \\
\hline \multirow[t]{2}{*}{ Stent size, Fr/cm } & $6 / 26(n=15)$ & $6 / 10(n=9)$ & \\
\hline & $4.5 / 26(n=2)$ & $4.8 / 10(n=1)$ & \\
\hline Median indwelling time, d (IQR) & $36(24.5-66)$ & $29.5(26-37)$ & 0.4 \\
\hline
\end{tabular}

IQR, interquartile range; UTI, urinary tract infection; yr, year; d, day.

$P$ values are calculated using the Mann-Whitney U-test for continuous data (age, indwelling time), and the Fisher's exact test for categorical data (gender, UTI).

\section{Encrustation Over Time}

The total encrustation volume ratios (TEVRs) from stone patients were significantly different $(\mathrm{p}=0.02)$ between indwelling times $<42 \mathrm{~d}$ (median: 0.45 , IQR: 0.10-0.80) and $\geq$ $42 \mathrm{~d}$ (median: 5.9, IQR: 3.8-26). Further comparisons of the encrustation volume ratios (EVRs) in each section (Figure 3A) revealed that encrustations increased significantly in the renal pigtail $(p=0.002)$ and the distal straight part $(p=0.01)$. The EVR in the proximal straight part also showed considerable increase over six weeks but did not reach statistical significance $(p=0.1)$. In the transplant group no significant difference was found between short indwelling time $(<28 \mathrm{~d})$ and long indwelling time ( $\geq 28 \mathrm{~d}$ ) groups, although the median EVRs were higher in both pigtails (Figure 3B). The comparison was also made between stents from stone and transplant groups with indwelling times $<42 \mathrm{~d}$ (Figure 3C) and no differences were found in $\operatorname{EVR}(\mathrm{p}>0.05$ in all sections) or TEVR $(\mathrm{p}=0.2)$.

\section{Most Encrusted Stent Region}

Subsequent comparisons of encrustation risk levels (ERLs) were made between stone and transplant groups for each stent section (Figure 3D). In both patient groups, the highest median ERLs were found in the pigtails with no significant difference between renal and bladder. Interestingly, in stone patients, the ERL of the proximal straight part was higher than that of the distal part ( $\mathrm{p}=$ 0.013). In transplant patients, however, no significant difference was found between the two straight parts. The ERLs of the pigtails were significantly higher than in the proximal $(p=0.022)$ and distal $(\mathrm{p}=0.014)$ straight parts, respectively. Comparison of ERLs between stone and transplant groups revealed a significant difference in the proximal straight part, with the higher ERL in the stone patients $(\mathrm{p}=0.007)$. Further data can be found in the Supplementary Material.

\section{DISCUSSION}

The segmentation method based on the CNN model U-Net (16) allowed us to evaluate luminal encrustation and stent volumes simultaneously with unparalleled accuracy. Once trained, the model can be applied to subsequent data sets without further tuning, and therefore reduces random error or bias imposed during the quantitative analyses. Moreover, to the best of the authors' knowledge, this study is the first to measure both the encrustation volume and the stent volume. As such, the encrustation volume per stent unit was derived, which is more representative than directly comparing the encrustation volumes, as was done in several previous studies $(13,14)$.

One observation in our study was that SHs were often the anchoring sites of encrustations even for stents with short indwelling times regardless of the patient group (Figure 2). The initial deposits of encrustations near SHs might be explained by recent in vitro experiments such that $\mathrm{SH}$ facilitate local urine flow stasis and promote particle accumulation in the neighboring regions (17-19). These initial deposits exacerbate the local encrustation process, causing severe stone burden near SHs, which has been previously reported in clinical studies $(11,20)$. The stony encrustation could compromise the stent tensile strength at the SHs, and eventually deteriorate into fractures $(11,20)$. In spite of the negative effects, stents with SHs should not be simply advised against as they are crucial in exchanging urine flow between the stent lumen and extraluminal spaces (between the stent and the ureter wall) in case of obstruction. Further studies are required to fully evaluate their efficacy in order to give clinical suggestions.

Another observation was that that encrustation levels did not differ between patient groups in the first six weeks (Figure 3C), but stone patients had a higher tendency to build up encrustations over prolonged indwelling time than transplant patients (Figures 3A, B). The higher tendency could be explained by the fact that stone patients have supersaturated urine. The similar encrustation levels in the first six weeks, however, would require further studies to fully clarify, as the early stages of encrustation involve biological processes such as the formation of conditioning film and biofilms, which might have an impact on the encrustation levels in both patient groups in the long term.

The highest median ERLs were observed in the pigtails in both patient groups, suggesting higher risks of excessive encrustations per stent unit. The pigtails at both ends exhibited high median in ERL and did not differ from each other, so the risk seemed 

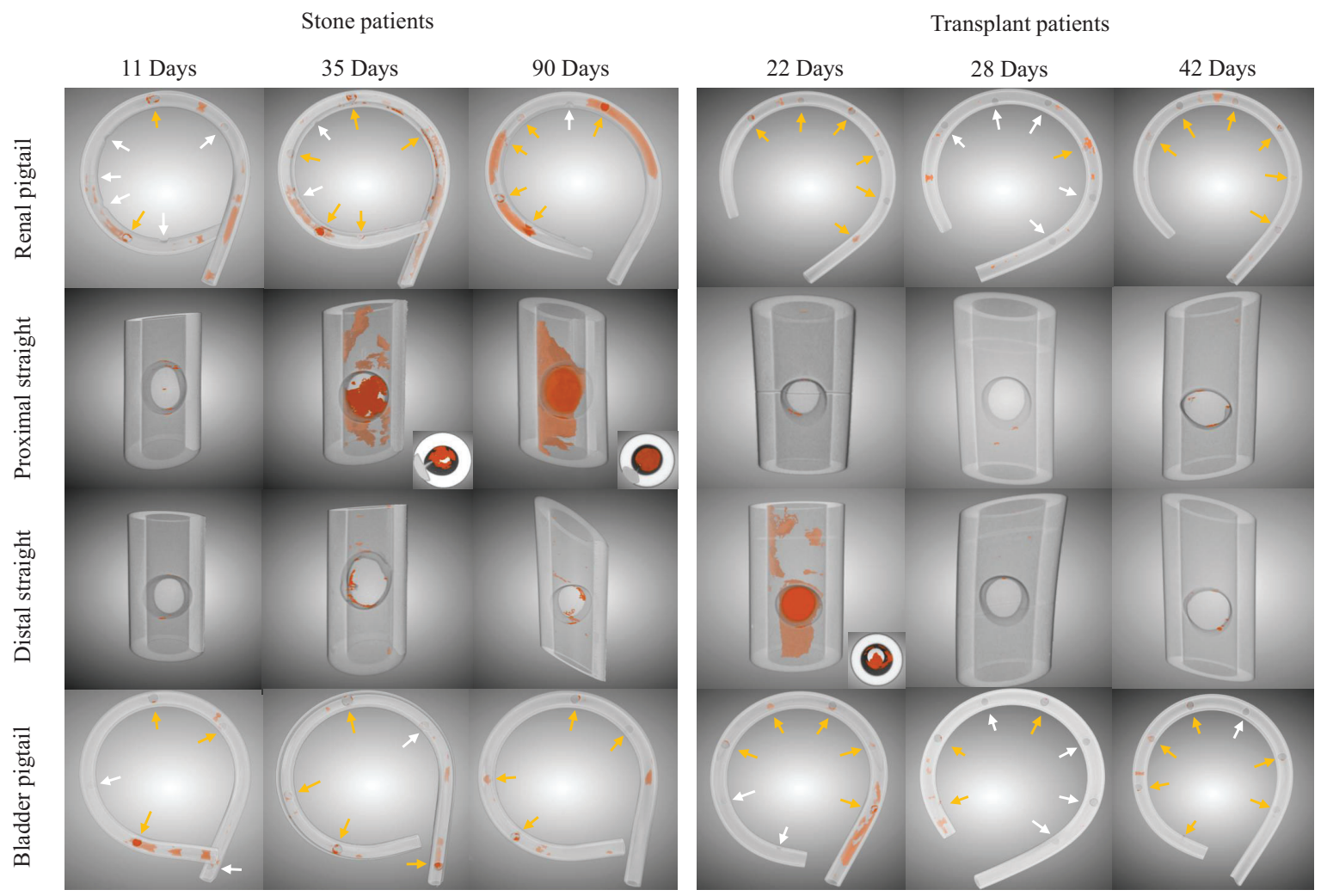

FIGURE 2 | Examples of luminal encrustations (orange) in the renal pigtail, proximal straight part, distal straight part, and bladder pigtail of the stents from stone and transplant patient groups. Stents are rendered semi-transparent for better visualization. Side hole locations on the pigtails are marked by arrows, where yellow arrows indicate clear evidence of encrustations and white arrows suggest otherwise. Cross sectional insets are given for visually significant encrustations to show the blockage level in the stent lumen.

equivalent in the renal and bladder ends. Since our results were based on encrustation volume per stent unit, it seems that shorter pigtails are preferrable to alleviate encrustations. The shorter length may also reduce other stent associated complications since stents crossing the bladder midline has been reported to cause more pain and urinary symptoms (21).

Nonetheless, the double pigtail or multi-coil stents are less prone to migration, which is also a significant complication of indwelling stent (22). The risks of encrustation and migration with shorter pigtail must be balanced. Further assessment on the encrustation level and urinary symptoms against pigtail length may offer valuable perspectives on the clinical choice of stents.

In practice, proximal stone burden has been associated with multiple surgical complications (23), and accurate determination of the stent section most susceptible to encrustations has been an active topic of discussion. Previous studies on stent encrustation patterns mainly focused on stone patients. While some suggested the renal pigtail as the most susceptible section followed by the bladder pigtail $(4,11,13)$, others reported that the two pigtails were most and equally susceptible (9), or that the distal part of stent (12) was most encrusted. The recent study using $\mu$-CT (14) also suggested the stent's shaft body (the entire straight part) as the most susceptible region. For one thing, the disputed conclusions can be attributed to the lack of quantitative tools to accurately measure the encrustation volumes. For another, the fact that there were no significant differences between certain comparisons (as demonstrated in Figure 3) should also be acknowledged, such that qualitative observations can be biased. By identifying the most encrusted stent region, we hope to guide further studies to address the most problematic regions of current stents, offering possible ideas for subsequent optimizations. The encrustation volume per stent unit presented in the current study might be adopted for subsequent works, which offers more meaningful insights than direct comparison of encrustation volumes.

In contrast to stone patients, quantitative evaluation of stent encrustation patterns in transplant patients are lacking. Following kidney transplantation, stents are usually placed to prevent strictures or urine leakage. These prophylactic stents are much shorter than those in stone patients since the allograft ureter is kept short to ensure a good blood supply. Consequently, higher grades of vesicoureteral reflux (VUR) in transplant patients are common as urine reaches up to the renal pelvis 
A
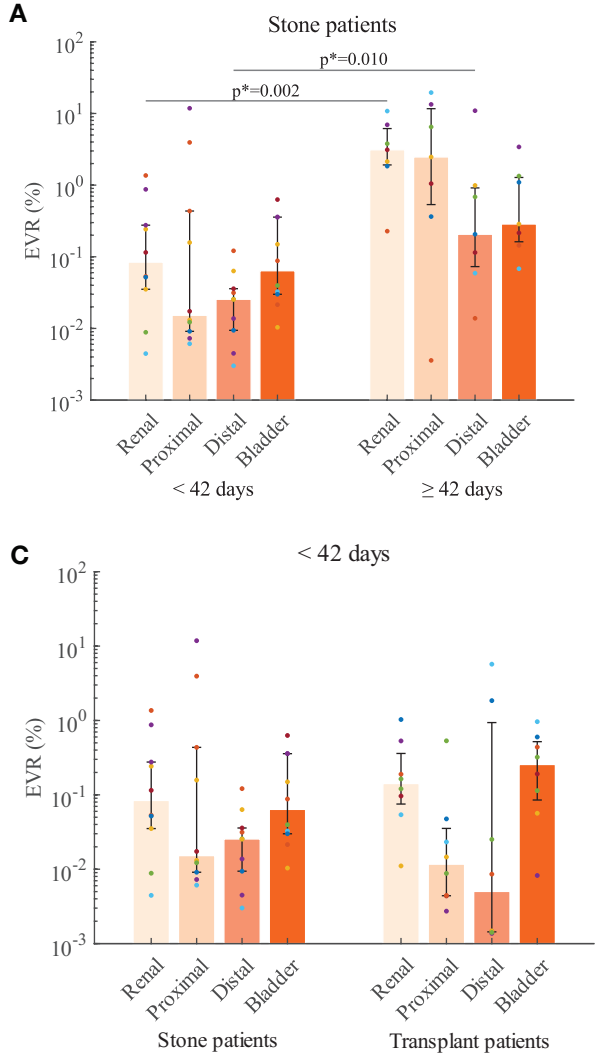

B
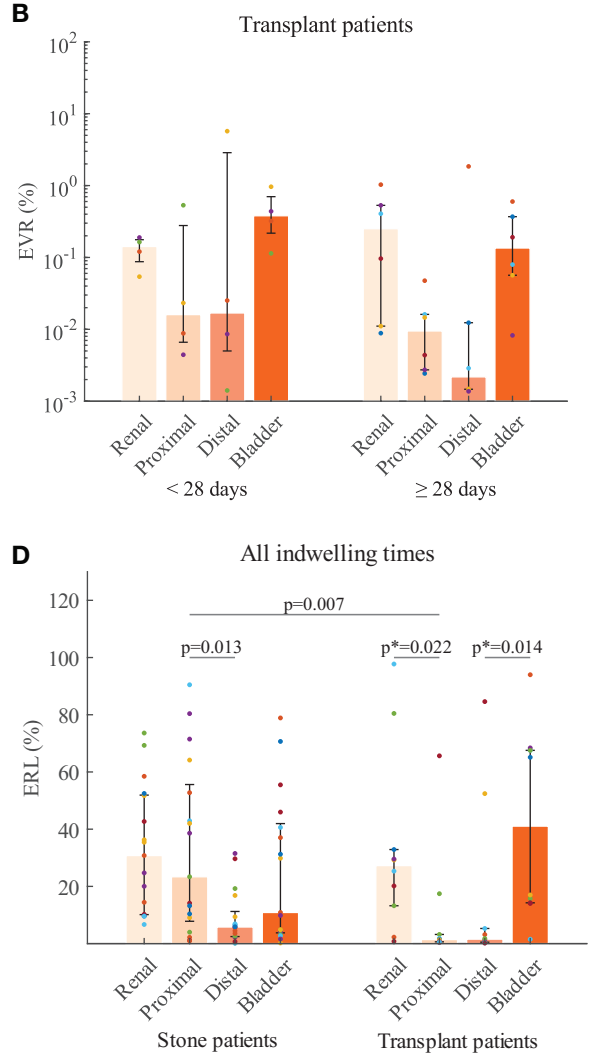

FIGURE 3 | Encrustation volume ratios (EVR) compared with respect to indwelling time for stone patients (A) and transplant patients (B). (C) EVR compared between patient groups with stent indwelling time $<42$ days. (D) Encrustation risk levels (ERL) from stone and transplant patients for each stent section. The median (bar) and interquartile range (error bars) are presented. Raw data from the same stent are coded by color (filled circles). P-values corrected by the Bonferroni-Holm method is indicated by the asteroid $\left(^{*}\right)$. Further data can be found in the Supplementary Material.

more easily (24). This retrograde flow might create a flushing effect, periodically stirring the deposits in the stent lumen, whereas in stone patients the VUR would not reach up to renal pelvis as easily since the stents are often longer. This might explain the higher encrustation level in the proximal straight part in stone patients (Figure 3D).

Moreover, stent implantation significantly impedes the peristalsis of the ureter (25), and thus the urine transport from kidney to bladder is largely passive. The openings at each end of the stent lumen (usually with internal diameter of $1 \mathrm{~mm}$ ) create significant hydraulic resistance to the urine flow so local stasis are expected in the pigtails. In the stent shaft, since the hydraulic resistance is proportional to the tube length, the shorter stent in transplant patients could better facilitate urine flows in the stent lumen, either from kidney to bladder or in the presence of VUR. As such, on the basis that encrustations are regulated by the local fluid mechanical characteristics $(17,26)$, the luminal encrustation levels along the stent are influenced by both the forward and retrograde urine flows, co-creating the different patterns presented in Figure 3.
Limitations of this study include the limited sample size, which did not allow for further subgroup analysis. Discussions regarding the biological aspects of the encrustation process were therefore missing. An extended study focusing on the correlation between specific complications and degree of encrustation would be highly desirable. Also, by separating the stent into four parts, the central part of the stent shaft was omitted. It might be interesting to assess the distribution of encrustations along the entire stent body. Nevertheless, the current study offers the first data connecting urinary flow dynamics and quantitative encrustation levels. Further studies on the specific urine flow conditions at each part of the stent could help elucidate the process of encrustations in stented native or allograft ureters.

As a closing remark, the semantic segmentation approach delivered accurate and intuitive results and is applicable to other image analysis tasks in urology. Our results highlighted the similarities and differences in stent encrustation patterns between stone and renal transplant groups, and possible explanations were discussed. Further investigations in both engineering and clinical disciplines are necessary to fully 
understand the dynamics of encrustations in order to develop the "perfect stent".

\section{DATA AVAILABILITY STATEMENT}

The original contributions presented in the study are included in the article/Supplementary Material. Further inquiries can be directed to the corresponding author.

\section{ETHICS STATEMENT}

Ethical review and approval was not required for the study on human participants in accordance with the local legislation and institutional requirements. Written informed consent for participation was not required for this study in accordance with the national legislation and the institutional requirements.

\section{AUTHOR CONTRIBUTIONS}

Conception and design: FB, FC, DO, and SZ. Acquisition of data: PA, FB, BK, FS, and SZ. Analysis and interpretation of data: PA and SZ. Figure preparation: PA, SZ. Drafting of the manuscript:

\section{REFERENCES}

1. Bibby LM, Wiseman OJ. Double JJ Ureteral Stenting: Encrustation and Tolerability. Eur Urol Focus (2020) 7(1):7-8. doi: 10.1016/j.euf.2020.08.014

2. Tomer N, Garden E, Small A, Palese M. Ureteral Stent Encrustation: Epidemiology, Pathophysiology, Management and Current Technology. J Urol (2021) 205(1):68-77. doi: 10.1097/JU.0000000000001343

3. El-Faqih SR, Shamsuddin AB, Chakrabarti A, Atassi R, Kardar AH, Osman MK, et al. Polyurethane Internal Ureteral Stents in Treatment of Stone Patients: Morbidity Related to Indwelling Times. J Urol (1991) 146 (6):1487-91. doi: 10.1016/S0022-5347(17)38146-6

4. Kawahara $\mathrm{T}$, Ito $\mathrm{H}$, Terao $\mathrm{H}$, Yoshida M, Matsuzaki J. Ureteral Stent Encrustation, Incrustation, and Coloring: Morbidity Related to Indwelling Times. J Endourol (2011) 26(2):178-82. doi: 10.1089/end.2011.0385

5. Tavakoli A, Surange RS, Pearson RC, Parrott NR, Augustine T, Riad HN. Impact of Stents on Urological Complications and Health Care Expenditure in Renal Transplant Recipients: Results of a Prospective, Randomized Clinical Trial. J Urol (2007) 177(6):2260-4. doi: 10.1016/j.juro. 2007.01.152

6. Thompson ER, Hosgood SA, Nicholson ML, Wilson CH. Early Versus Late Ureteric Stent Removal After Kidney Transplantation. Cochrane Database Syst Rev (2018) 1:1-27. doi: 10.1002/14651858.CD011455.pub2

7. Visser IJ, van der Staaij JPT, Muthusamy A, Willicombe M, Lafranca JA, Dor FJMF. Timing of Ureteric Stent Removal and Occurrence of Urological Complications After Kidney Transplantation: A Systematic Review and Meta-Analysis. J Clin Med (2019) 8(5):1-15. doi: 10.3390/jcm8050689

8. Wilson CH, Rix DA, Manas DM. Routine Intraoperative Ureteric Stenting for Kidney Transplant Recipients. Cochrane Database Syst Rev (2013) 6:1-24. doi: 10.1002/14651858.CD004925.pub3

9. Arkusz K, Pasik K, Halinski A, Halinski A. Surface Analysis of Ureteral Stent Before and After Implantation in the Bodies of Child Patients. Urolithiasis (2020) 49:83-92. doi: 10.1007/s00240-020-01211-9

10. Acosta-Miranda AM, Milner J, Turk TMT. The FECal Double-J: A Simplified Approach in the Management of Encrusted and Retained Ureteral Stents. J Endourol (2009) 23(3):409-15. doi: 10.1089/end.2008.0214
SZ. Statistical analysis: AH and SZ. All authors contributed to the article and approved the submitted version.

\section{FUNDING}

The work is supported by the European Cooperation in Science and Technology (ENIUS COST action, grant number CA16217) and the Swiss National Science Foundation (SNSF, grant number IZCOZ0_182966).

\section{ACKNOWLEDGMENTS}

SZ acknowledges Mr. Michael Indermaur from the Musculoskeletal Biomechanics group at the University of Bern for his support in operating the Micro-CT scanner, and Mr. Emile Talon for his contribution in the preliminary study.

\section{SUPPLEMENTARY MATERIAL}

The Supplementary Material for this article can be found online at: https://www.frontiersin.org/articles/10.3389/fruro.2022. 836563/full\#supplementary-material

11. Singh I, Gupta NP, Hemal AK, Aron M, Seth A, Dogra PN. Severely Encrusted Polyurethane Ureteral Stents: Management and Analysis of Potential Risk Factors. Urology (2001) 58(4):526-31. doi: 10.1016/S00904295(01)01317-6

12. Rana AM, Sabooh A. Management Strategies and Results for Severely Encrusted Retained Ureteral Stents. J Endourol (2007) 21(6):628-32. doi: 10.1089/end.2006.0250

13. Sighinolfi MC, Sighinolfi GP, Galli E, Micali S, Ferrari N, Mofferdin A, et al. Chemical and Mineralogical Analysis of Ureteral Stent Encrustation and Associated Risk Factors. Urology (2015) 86(4):703-6. doi: 10.1016/ j.urology.2015.05.015

14. Yoshida T, Takemoto K, Sakata Y, Matsuzaki T, Koito Y, Yamashita S, et al. A Randomized Clinical Trial Evaluating the Short-Term Results of Ureteral Stent Encrustation in Urolithiasis Patients Undergoing Ureteroscopy: MicroComputed Tomography Evaluation. Sci Rep (2021) 11(1):10337. doi: 10.1038/ s41598-021-89808-x

15. Vandenbroucke JP, von Elm E, Altman DG, Gøtzsche PC, Mulrow CD, Pocock SJ, et al. Strengthening the Reporting of Observational Studies in Epidemiology (STROBE): Explanation and Elaboration. PloS Med (2007) 4 (10):e297. doi: 10.1371/journal.pmed.0040297

16. Ronneberger O, Fischer P, Brox T, eds. U-Net: Convolutional Networks for Biomedical Image Segmentation. In: 18th International Conference on Medical Image Computing and Computer-Assisted Intervention (MICCAI 2015), 2015 October 5-9. Munich, Germany: Springer International Publishing (2015). doi: 10.1007/978-3-319-24574-4_28

17. Mosayyebi A, Yue QY, Somani BK, Zhang X, Manes C, Carugo D. Particle Accumulation in Ureteral Stents Is Governed by Fluid Dynamics: In Vitro Study Using a "Stent-On-Chip" Model. J Endourol (2018) 32(7):639-46. doi: 10.1089/end.2017.0946

18. Clavica F, Zhao X, ElMahdy M, Drake MJ, Zhang X, Carugo D. Investigating the Flow Dynamics in the Obstructed and Stented Ureter by Means of a Biomimetic Artificial Model. Proceedings of the 18th International Conference on Miniaturized Systems for Chemistry and Life Sciences, MicroTAS. PloS One (2014) 9(2):e87433. doi: 10.1371/journal. pone. 0087433 
19. D Carugo, X Zhang, JM Drake, F Clavica, eds. Formation and Characteristics of Laminar Vortices in Microscale Environments Within an Obstructed and Stented Ureter: A Computational Study. In: Proceedings of the 18th International Conference on Miniaturized Systems for Chemistry and Life Sciences, MicroTAS. San Antonio, Texas, USA: MicroTAS.

20. Zisman A, Siegel YI, Siegmann A, Lindner A. Spontaneous Ureteral Stent Fragmentation. J Urol (1995) 153(3):718-21. doi: 10.1016/S0022-5347(01) 67697-3

21. Taguchi M, Yoshida K, Sugi M, Matsuda T, Kinoshita H. A Ureteral Stent Crossing the Bladder Midline Leads to Worse Urinary Symptoms. Cent Eur J Urol (2017) 70(4):412-7. doi: 10.5173/ceju.2017.1533

22. Saltzman B. Ureteral Stents: Indications, Variations, and Complications. Urol Clin N Am (1988) 15(3):481-91. doi: 10.1016/S0094-0143(21)01594-9

23. Weedin JW, Coburn M, Link RE. The Impact of Proximal Stone Burden on the Management of Encrusted and Retained Ureteral Stents. J Urol (2011) 185 (2):542-7. doi: 10.1016/j.juro.2010.09.085

24. Ness D, Olsburgh J. UTI in Kidney Transplant. World J Urol (2020) 38(1):818. doi: 10.1007/s00345-019-02742-6

25. Mosli Hisham A, Farsi Hasan MA, Al-Zimaity Mohammed F, Saleh Tarik R, AlZamzami Mokhtar M. Vesicoureteral Reflux in Patients With Double Pigtail Stents. J Urol (1991) 146(4):966-9. doi: 10.1016/S0022-5347(17)37976-4
26. Zheng S, Carugo D, Mosayyebi A, Turney B, Burkhard F, Lange D, et al. Fluid Mechanical Modeling of the Upper Urinary Tract. WIREs Mech Dis (2021) 13 (6):e01523. doi: 10.1002/wsbm.1523

Conflict of Interest: The authors declare that the research was conducted in the absence of any commercial or financial relationships that could be construed as a potential conflict of interest.

Publisher's Note: All claims expressed in this article are solely those of the authors and do not necessarily represent those of their affiliated organizations, or those of the publisher, the editors and the reviewers. Any product that may be evaluated in this article, or claim that may be made by its manufacturer, is not guaranteed or endorsed by the publisher.

Copyright (C) 2022 Zheng, Amado, Kiss, Stangl, Haeberlin, Sidler, Obrist, Burkhard and Clavica. This is an open-access article distributed under the terms of the Creative Commons Attribution License (CC BY). The use, distribution or reproduction in other forums is permitted, provided the original author(s) and the copyright owner(s) are credited and that the original publication in this journal is cited, in accordance with accepted academic practice. No use, distribution or reproduction is permitted which does not comply with these terms. 